\title{
Strong and Electromagnetic Decays of X(1835) as a Baryonium State
}

\author{
Yong-Liang Ma \\ Institute for Theoretical Physics, University of Tuebingen, D-72076 Tuebingen, Germany
}

(Dated: October 27, 2018)

\begin{abstract}
With the assumption that the recently observed $\mathrm{X}(1835)$ is a baryonium state we have studied the strong decays of $X(1835) \rightarrow \eta^{(\prime)} \pi^{+} \pi^{-}, \eta^{(\prime)} \pi^{0} \pi^{0}$ and the electromagnetic decay of $X(1835) \rightarrow 2 \gamma$ in the framework of effective Lagrangian formalism. In the present investigation we have included the contributions from the iso-singlet light scalar resonances but we have not included the isospin violating effect. Our result for the strong decay of $X(1835) \rightarrow \eta^{\prime} \pi^{+} \pi^{-}$is smaller than the observed data. The decay width for the radiative decay of $X(1835) \rightarrow 2 \gamma$ is consistent with the assumption that it decays through the glueball. In addition, the width for the strong decay of $X(1835) \rightarrow \eta \pi^{+} \pi^{-}$is larger than that of the strong decay of $X(1835) \rightarrow \eta^{\prime} \pi^{+} \pi^{-}$due to the large phase space and coupling constant $g_{N \bar{N} \eta}$. From our investigation, it is not possible to interpret $\mathrm{X}(1835)$ as a baryonium.

PACS numbers: 13.25.Jx,12.39.Mk, 13.40.Hq
\end{abstract}

\section{INTRODUCTION}

In 2005, the BES collaboration announced the observation of a resonant state termed $\mathrm{X}(1835)$ in the reaction $J / \psi \rightarrow \gamma X, X \rightarrow \eta^{\prime} \pi^{+} \pi^{-}[1]$. A fit to this resonance with the Breit-Wigner function yields the quantum number $J^{P C}=0^{-+}$and mass $M_{X}=(1833.7 \pm 6.1 \pm 2.7) \mathrm{MeV}$, width $\Gamma_{X}=$ $(67.7 \pm 20.3 \pm 7.7) \mathrm{MeV}$ and the product branching fraction $\operatorname{Br}(J / \psi \rightarrow \gamma X(1835)) \operatorname{Br}(X(1835) \rightarrow$ $\left.\eta^{\prime} \pi^{+} \pi^{-}\right)=(2.2 \pm 0.4$ (stat. $) \pm 0.4$ (syst. $\left.)\right) \times 10^{-4}$. Actually, without include the final state interaction, the parameters of this resonance have been fitted to be $M_{X} \simeq 1859_{-10}^{+3}$ (stat) ${ }_{-25}^{+5}$ (syst) $\mathrm{MeV}$ and the total width $\Gamma<30 \mathrm{MeV}$ in Ref. [2]. Since the discovery of the X(1835) state, many models have been proposed to explain its properties [3, 4, 5, 6, 7, 8, 9, 10, 11, 12, 13, 14, 15, 16, 17, 18, 19].

In the previous works, the $\mathrm{X}(1835)$ state has been conjectured to be a baryonium state $[3,4$, 5, 6, 7, 8, 9, 10, 11, 12, 13], pseudoscalar glueball state [14, 15, 16] and also a radial excitation of $\eta^{\prime}[17,18,19]$. Although there are many speculations, none of the above claims can be either confirmed or ruled out by the present experiments. In our present work, we have calculated the strong decays of $X(1835) \rightarrow \eta^{(\prime)} \pi^{+} \pi^{-}, \eta^{(\prime)} \pi^{0} \pi^{0}$ and radiative decay of $X(1835) \rightarrow 2 \gamma$ using the effective Lagrangian formalism by treating the $\mathrm{X}(1835)$ as a $N \bar{N}$ baryonium. This seems to be a reasonable approximation if one only considers the fact that the mass of the $\mathrm{X}(1835)$ is bit lower 
than the threshold energy of $p \bar{p}$ and $n \bar{n}$ (about $40 \mathrm{MeV}$ ). Our philosophy is that, assuming the $\mathrm{X}(1835)$ as a baryonium, if we can get the numerical results agree with the observed data the baryonium assumption is reasonable otherwise the baryonium picture can be ruled out, at least in this framework. The coupling of the $\mathrm{X}(1835)$ to its constituents can be described by the effective Lagrangian. The corresponding effective coupling constant $g_{X}$ is determined by the compositeness condition $Z=0$ which was earlier used by nuclear physicists [20, 21, 22] and is being widely used by particle physicists (see the references in 23]). We had applied the above method to study the newly observed charmed mesons [23, 24, 25] and their decay properties which we had obtained agreed with the observed data. We had also employed the above technique to predict the decay properties of the bottom-strange mesons [26]. In our present work, we have used a typical scale parameter $\Lambda_{X}$ to describe the finite size of the baryonium. The value of $\Lambda_{X}$ is fixed by considering the coupling constant $g_{X}$ is expected to be stable. For other interactions, we have used the phenomenological Lagrangian and have borrowed the relevant coupling constants from the existing literature. Using the above phenomenological approaches, we have analyzed the strong decays of $X(1835) \rightarrow \eta^{(\prime)} \pi^{+} \pi^{-}, \eta^{(\prime)} \pi^{0} \pi^{0}$ and radiative decay of $X(1835) \rightarrow 2 \gamma$. The result of the decay width of $X(1835) \rightarrow \eta^{\prime} \pi^{+} \pi^{-}$is much smaller than the observed data hence the $\mathrm{X}(1835)$ cannot be treated as a baryonium.

The paper is organized in the following way: In Section II we have calculated the effective coupling constant $g_{X}$ using the compositeness condition and have discussed the effective Lagrangian formalism employed in our calculation. In Section III we have calculated the strong decay widths of $X(1835) \rightarrow \eta^{(\prime)} \pi^{+} \pi^{-}$and radiative decay width of $X(1835) \rightarrow 2 \gamma$ using the effective coupling constant $g_{X}$ and effective Lagrangian proposed in Section II] In section IV the important results and conclusions have been given.

\section{THEORETICAL FRAMEWORK}

\section{A. Baryonium structure of the $\mathrm{X}(\mathbf{1 8 3 5})$ state}

In this section we give the formulation for the study of the $\mathrm{X}(1835)$ as a baryonium state which can be thought of as a $p \bar{p}(n \bar{n})$ bound state. As stated earlier, the mass of the $\mathrm{X}(1835)$ is around $40 \mathrm{MeV}$ less than the threshold of $p \bar{p}(n \bar{n})$. The quantum number of the $\mathrm{X}(1835)$ is assigned to be $J^{P C}=0^{-+}$, and its mass is predicted to be $m_{X}=1833.7 \mathrm{MeV}$ [1]. The effective Lagrangian 
describing the interaction between the $\mathrm{X}(1835)$ and its constituents is given by

$$
\mathcal{L}_{X(1835)}(x)=i g_{X} X(x) \int d y \Phi_{X}\left(y^{2}\right) \bar{N}\left(x+\frac{1}{2} y\right) \gamma_{5} N\left(x-\frac{1}{2} y\right)
$$

where the baryon doublet $N$ is defined as

$$
N=\left(\begin{array}{l}
p \\
n
\end{array}\right)
$$

The correlation function $\Phi_{X}$ characterizes the finite size of the $\mathrm{X}(1835)$ as a $N \bar{N}$ bound state and depends on the relative Jacobi coordinates $y$ and $x$. In the limit $\Phi_{X}\left(y^{2}\right) \rightarrow \delta^{4}(y)$, the interaction given by Eq. (1) becomes local. The Fourier transform of the correlation function $\Phi_{X}\left(y^{2}\right)$ is

$$
\Phi_{X}\left(y^{2}\right)=\int \frac{d^{4} p}{(2 \pi)^{4}} e^{-i p \cdot y} \tilde{\Phi}_{X}\left(-p^{2}\right)
$$

In following calculation, an explicit form of $\tilde{\Phi}_{X}$ has been used. The choice of $\tilde{\Phi}_{X}$ should be such that it falls off sufficiently fast in the ultraviolet region of Euclidean space to render the Feynman diagrams finite in the UV region. In this sense, one can also regard $\tilde{\Phi}_{X}$ as a regulator for the loop integral. In our work, we have chosen the Gaussian form for $\tilde{\Phi}_{X}$

$$
\tilde{\Phi}_{X}\left(p_{E}^{2}\right)=\exp \left(-p_{E}^{2} / \Lambda_{X}^{2}\right)
$$

where $p_{E}$ is the Euclidean Jacobi momentum. Here $\Lambda_{X}$ is a size parameter which parameterizes the distribution of $N$ and $\bar{N}$ baryons inside the $\mathrm{X}(1835)$ baryonium.

The coupling constant $g_{X}$ is determined by the compositeness condition [20, 21, 22] which implies that the renormalization constant of the hadron wave function is set to zero

$$
Z_{X}=1-\Sigma_{X}^{\prime}\left(m_{X}^{2}\right)=0
$$

Here, $\Sigma_{X}^{\prime}\left(m_{X}^{2}\right)=g_{X}^{2} \Pi_{X}^{\prime}\left(m_{X}^{2}\right)$ is the derivative of the mass operator $\Sigma_{X}$ which is represented by the diagrams in Fig. 1 given below.
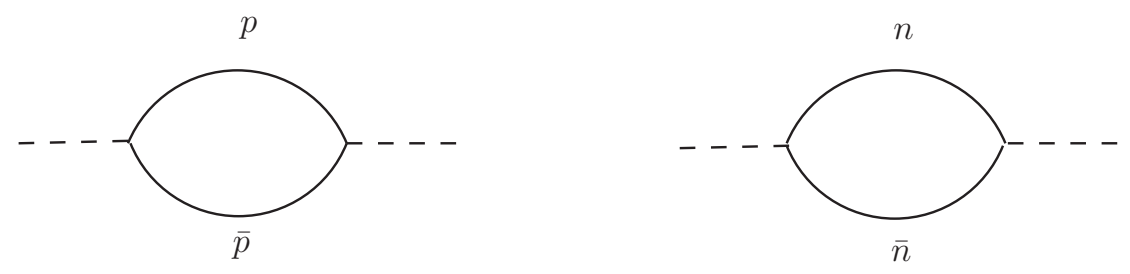

FIG. 1: Mass operator of $\mathrm{X}(1835)$ as a baryonium state. 
The compositeness condition can be understood in the following: The renormalization constant $Z_{X}^{1 / 2}$ can be interpreted as the matrix element between the physical state $\mathrm{X}(1835)$ and corresponding bare state $X_{0}(1835)$, i.e., $\left\langle 0\left|X_{0}(1835)\right| X(1835)\right\rangle=Z_{X}^{1 / 2}\langle 0|X(1835)| X(1835)\rangle=Z_{X}^{1 / 2}$, so that $Z_{X}=0$ means that the physical state should not be a function of the corresponding bare state which means that the physical state is a bound state. In our present work, the $\mathrm{X}(1835)$ is a bound state of $p \bar{p}(n \bar{n})$. In this sense, the compositeness condition excludes the possibility of the processes involving the $\mathrm{X}(1835)$ as an initial or a final state since each external $\mathrm{X}(1835)$ contributes a factor $Z_{X}^{1 / 2}$ to the relevant matrix elements. In addition, because of the interaction between the $\mathrm{X}(1835)$ and its constituents, the mass and wave function of the $\mathrm{X}(1835)$ have to be renormalized.

Following Eq. (2) the coupling constant $g_{X}$ can be expressed as

$$
\frac{1}{g_{X}^{2}}=\frac{1}{4 \pi^{2}} \int_{0}^{\infty} d \alpha \int_{0}^{1} d x \frac{1}{(1+\alpha)^{2}} \frac{d}{d \mu_{X}^{2}}\left\{\tilde{\Phi}\left(z_{1}\right)+\alpha \mu_{X}^{2} \tilde{\Phi}\left(z_{2}\right)\right\}
$$

where $\alpha$ and $x$ are both Feynman parameters and

$$
\begin{aligned}
z_{1} & =\alpha m_{p}^{2}-\frac{\alpha}{4(1+\alpha)} m_{X}^{2} \\
z_{2} & =\alpha m_{p}^{2}-\frac{\alpha+4 \alpha^{2} x(1-x)}{4(1+\alpha)} m_{X}^{2} \\
\mu_{X}^{2} & =m_{X}^{2} / \Lambda_{X}^{2}
\end{aligned}
$$

$x$ and $\alpha$ are both Feynman parameters. In deriving the expression (3), we have ignored the mass difference between proton and neutron and expressed the coupling constant $g_{X}$ in terms of the proton mass. To get the numerical result of $g_{X}$, we use $m_{X}=1833.7 \mathrm{MeV}[1], m_{p}=$ 938.272 MeV [27] and vary the scale parameter $\Lambda_{X}$ from $1.0 \mathrm{GeV}$ to $5.0 \mathrm{GeV}$. In Fig. 2 we show the $\Lambda_{X}$ dependence of the effective coupling constant $g_{X}$.

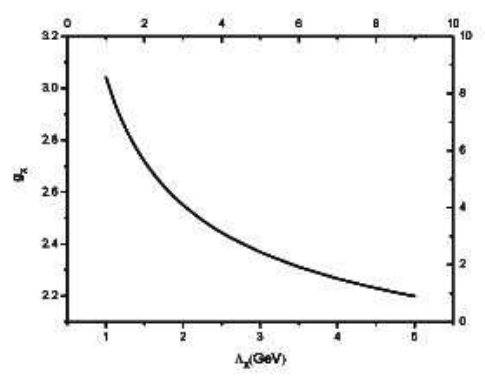

FIG. 2: The $\Lambda_{X}$ dependence of the coupling constant $g_{X}$. 
Concerning that $g_{X}$ is expected to be stable against $\Lambda_{X}$, we choose the region of $\Lambda_{X}$ as $2.0 \mathrm{GeV} \leq \Lambda_{X} \leq 3.0 \mathrm{GeV}$ and get the coupling constant to be in the range $g_{X}=2.37-2.55$. Comparing our present result with that given in Ref. [12] where this coupling constant was estimated from experimental branching ratio of the $\mathrm{X}(1835)$ to $p \bar{p}$ decay in radiative decay of $J / \psi$ (by considering that $X \rightarrow p \bar{p}$ occurs from the tail of its mass distribution and the value was found to be, $\left|g_{X p \bar{p}}\right| \simeq 3.5$ ), we conclude our result agrees with the result given there. In fact, using $\mathrm{BR}(X \rightarrow p \bar{p}) \sim(0.04-0.14)$ assuming $\Gamma_{X}<30 \mathrm{MeV}$ [2] that Ref. [12] adopted, one can get $g_{X p \bar{p}}=2.2-4.1$. In addition, our conclusion is also consistent with that of Ref. [15] which was based on the glueball assumption. Expressing the coupling constant $g_{X \bar{p}}$ in terms of $g_{X_{g}}$ which is the coupling constant between the $\mathrm{X}(1835)$ and glueball, one can get $g_{X p \bar{p}}=2.47-4.67$.

\section{B. Effective Lagrangian for strong and electromagnetic decays of $\mathrm{X}(\mathbf{1 8 3 5})$}

In this section, we have discussed the effective Lagrangian for the calculation of the strong decays of $X(1835) \rightarrow \eta^{(\prime)} \pi^{+} \pi^{-}$and electromagnetic decay of $X(1835) \rightarrow 2 \gamma$. The effective lagrangian can be divided into two parts, the free part $\mathcal{L}_{\text {free }}$ and the interaction part $\mathcal{L}_{\text {int }}$. It should be noted that the electromagnetic interaction can be obtained by the minimal substitution (i.e., replacing the derivative operator $\partial_{\mu}$ of the charged particle with the covariant one $D_{\mu}=\partial_{\mu}-i e Q A_{\mu}$ with $Q$ as the charge of the relevant particle). For the free Lagrangian, it involves states with quantum numbers $J^{P}=\frac{1}{2}^{+}, 0^{-}, 0^{+}$and $1^{-}$.

$$
\mathcal{L}_{\text {free }}=\mathcal{L}_{\text {free }}^{N}+\mathcal{L}_{\text {free }}^{P}+\mathcal{L}_{\text {free }}^{S}+\mathcal{L}_{\text {free }}^{V}
$$

where

$$
\begin{aligned}
\mathcal{L}_{\text {free }}^{N}= & \bar{N}\left(i \not \supset-m_{N}\right) N \\
\mathcal{L}_{\text {free }}^{P}= & -\frac{1}{2} X(1835)\left(\square+m_{X}^{2}\right) X(1835) \\
& -\frac{1}{2} \vec{\pi}(x)\left(\square+m_{\pi}^{2}\right) \vec{\pi}(x)-\frac{1}{2} \eta(x)\left(\square+m_{\eta^{\prime}}^{2}\right) \eta(x)-\frac{1}{2} \eta^{\prime}(x)\left(\square+m_{\eta^{\prime}}^{2}\right) \eta^{\prime}(x) \\
\mathcal{L}_{\text {free }}^{S}= & -\frac{1}{2} \sigma\left(\square+m_{\sigma}^{2}\right) \sigma-\frac{1}{2} f_{0}\left(\square+m_{f_{0}}^{2}\right) f_{0} \\
\mathcal{L}_{\text {free }}^{V}= & -\frac{1}{4} F_{\mu \nu} F_{\mu \nu}
\end{aligned}
$$

with $F_{\mu \nu}=\partial_{\mu} A_{\nu}-\partial_{\nu} A_{\mu}$ as the field tensor of photon and $\square \equiv \partial_{\mu} \partial^{\mu}$. For computing the decays of the $\mathrm{X}(1835)$, we have treated the masses of proton and neutron and the masses of the triplet pions to be the same [1, 27]

$$
m_{X}=1833.7 \mathrm{MeV} ; \quad m_{n}=m_{p}=938.27203 \mathrm{MeV}
$$




$$
m_{\pi^{0}}=m_{\pi}^{ \pm}=139.57018 \mathrm{MeV} ; m_{\eta}=547.51 \mathrm{MeV} ; m_{\eta^{\prime}}=957.78 \mathrm{MeV}
$$

while for the masses and widths of scalar mesons, we have adopted [28]

$$
m_{\sigma}=550 \mathrm{MeV} ; \quad \Gamma_{\sigma}=370 \mathrm{MeV} ; \quad m_{f_{0}}=980 \mathrm{MeV} ; \quad \Gamma_{f_{0}}=64.6 \mathrm{MeV}
$$

In the following calculation, we have included the finite width effects of the scalar mesons, that is, we have written the scalar meson propagators as

$$
\tilde{D}_{S}(k)=\frac{i}{k^{2}-m_{S}^{2}+i m_{S} \Gamma_{S}}
$$

The interaction Lagrangian $\mathcal{L}_{\text {int }}$ used in our calculation has two parts, the strong part $\mathcal{L}_{\text {int }}^{\text {str }}$ and the electromagnetic part $\mathcal{L}_{\text {int }}^{\text {em }}$

$$
\mathcal{L}_{\text {int }}=\mathcal{L}_{\text {int }}^{\text {str }}+\mathcal{L}_{\text {int }}^{\text {em }}
$$

For the strong interaction Lagrangian we have $\mathcal{L}_{N \bar{N} X}$ (X-nucleon-nucleon interaction), $\mathcal{L}_{N \bar{N} P}$ (pseudoscalar-nucleon-nucleon interaction), $\mathcal{L}_{N \bar{N} S}$ (scalar-nucleon-nucleon interaction) and $\mathcal{L}_{S P P}$ (scalar-pseudoscalar-pseudoscalar interaction)

$$
\mathcal{L}_{\text {int }}^{\text {str }}=\mathcal{L}_{N \bar{N} X}+\mathcal{L}_{N \bar{N} P}+\mathcal{L}_{N \bar{N} S}+\mathcal{L}_{S P P}
$$

The effective Lagrangian $\mathcal{L}_{N \bar{N} X}$ was given in Eq. (11) and $\mathcal{L}_{N \bar{N} P}$ and $\mathcal{L}_{N \bar{N} S}$ can be expressed as

$$
\begin{aligned}
\mathcal{L}_{N \bar{N} P} & =\frac{1}{2 m} g_{N \bar{N} \pi} \bar{N} \gamma_{\mu} \gamma_{5} \vec{\tau} N \partial_{\mu} \vec{\pi}+\frac{1}{2 m} g_{N \bar{N} \eta} \bar{N} \gamma_{\mu} \gamma_{5} N \partial_{\mu} \eta+\frac{1}{2 m} g_{N \bar{N} \eta^{\prime}} \bar{N} \gamma_{\mu} \gamma_{5} N \partial_{\mu} \eta^{\prime} \\
\mathcal{L}_{N \bar{N} S} & =g_{N \bar{N} S} \bar{N} N S \\
\mathcal{L}_{S P P} & =-\frac{\gamma_{\sigma \pi \pi}}{\sqrt{2}} \sigma \partial_{\mu} \vec{\pi} \cdot \partial_{\mu} \vec{\pi}-\frac{\gamma_{f_{0} \pi \pi}}{\sqrt{2}} f_{0} \partial_{\mu} \vec{\pi} \cdot \partial_{\mu} \vec{\pi}
\end{aligned}
$$

where $S$ is the scalar meson ( $\sigma$ and $f_{0}$ in our problem) and $\pi$ is the pseudoscalar meson matrix

$$
\pi=\sum_{i=1}^{3} \pi^{i} \tau^{i}=\left(\begin{array}{cc}
\pi^{0} & \sqrt{2} \pi^{+} \\
\sqrt{2} \pi^{-} & -\pi^{0}
\end{array}\right)
$$

The coupling constants $g_{N \bar{N} \pi}, g_{N \bar{N} \eta}$ and $g_{N \bar{N} \eta^{\prime}}$ were determined via the $J / \psi$ hadronic decay [29, 30] while $g_{N \bar{N} S}$ was yielded by fitting the theoretical results of $N N$ scattering with the observed data [31]

$$
\begin{aligned}
\left(g_{N \bar{N} \pi}\right)^{2} /(4 \pi) & \simeq 14.8 \\
\left(g_{N \bar{N} \eta} / g_{N \bar{N} \pi}\right)^{2} & \simeq 3.90625 \times 10^{-3} \\
\left(g_{N \bar{N} \eta^{\prime}} / g_{N \bar{N} \pi}\right)^{2} & \simeq 2.5 \times 10^{-3}
\end{aligned}
$$




$$
\left(g_{N \bar{N} S}\right)^{2} /(4 \pi) \simeq 5.69
$$

The scalar-pseudoscalar-coupling constant $\gamma_{S P P}$ was given in Ref. [28]

$$
\gamma_{\sigma \pi \pi}=7.27 \mathrm{GeV}^{-1} ; \quad \gamma_{f_{0} \pi \pi}=1.47 \mathrm{GeV}^{-1}
$$

For the electromagnetic interaction Lagrangian $\mathcal{L}_{\text {int }}^{\text {em }}$ used in our calculation, it has two parts: (i) is from the gauge of the charged free nucleon Lagrangian, and (ii) is from the gauge of the nonlocal interaction

$$
\mathcal{L}_{\text {int }}^{\mathrm{em}}=\mathcal{L}_{\text {int }}^{\mathrm{em}(\mathrm{i})}+\mathcal{L}_{\text {int }}^{\mathrm{em}(\mathrm{ii})}
$$

where

$$
\begin{aligned}
\mathcal{L}_{\text {int }}^{\mathrm{em}(\mathrm{i})} & =e A_{\mu} \bar{N} \gamma_{\mu} \frac{1+\tau_{3}}{2} N \\
\mathcal{L}_{\text {int }}^{\mathrm{em}(\mathrm{ii})} & =i g_{X} X(x) \int d y \Phi_{X}\left(y^{2}\right)\left\{e^{i e I\left(x+\frac{1}{2} y, x-\frac{1}{2} y ; P\right)} \bar{p}\left(x+\frac{1}{2} y\right) \gamma_{5} p\left(x-\frac{1}{2} y\right)\right\}
\end{aligned}
$$

where the Wilson line $I(x, y, P)$ is defined as

$$
I(x, y ; P)=\int_{y}^{x} d z_{\mu} A^{\mu}(z)
$$

To derive the Feynman rules for photons, we require the derivative of $I(x, y ; P)$. For this we have used the path-independent prescription as suggested in Ref. 32, 33] which implies that the derivative of $I(x, y ; P)$ does not depend on the path $P$ originally used in the definition. Also in our calculation of $X(1835) \rightarrow 2 \gamma$, in principle we should expand the above expression to the second order but the diagram with photons from this vertex does not contribute since the trace of gamma matrices vanish.

\section{STRONG AND ELECTROMAGNETIC DECAYS}

Having discussed the effective coupling constant $g_{X}$ and the effective Lagrangian, we are in the position to calculate the decay properties of the $\mathrm{X}(1835)$. In this section, we have calculated the strong decays of $X(1835) \rightarrow \eta^{(\prime)} \pi^{+} \pi^{-}$and also the radiative decay of $X(1835) \rightarrow 2 \gamma$.

\section{A. Strong decays of $X(1835) \rightarrow \eta^{(\prime)} \pi^{+} \pi^{-}$}

For the strong decays of $X(1835) \rightarrow \eta^{(\prime)} \pi^{+} \pi^{-}$, the Feynman diagrams of Fig. 3 and Fig. 4 contribute. All the diagrams listed in Fig. 3 are from the one-pseudoscalar meson-nucleon-nucleon 
vertex while the diagrams listed in Fig. 4 are from the scalar resonance contributions. For the isospin symmetric case following relations among matrix elements exist

$$
i M_{(A)}=i M_{(D)} ; \quad i M_{(B)}=i M_{(E)} ; \quad i M_{(C)}=i M_{(F)}
$$

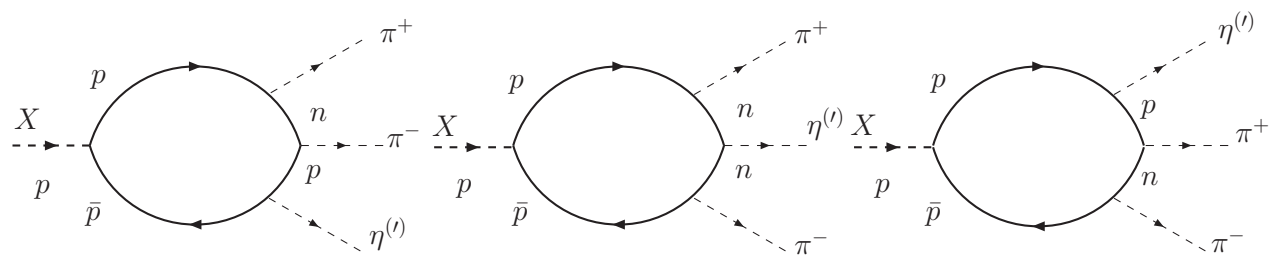

$(A)$

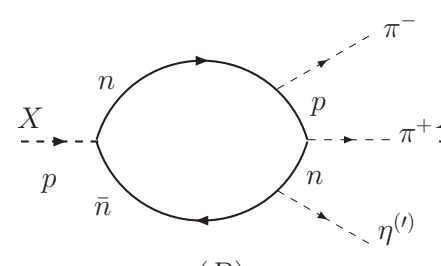

(D)

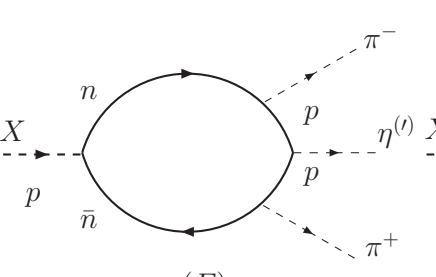

$(E)$

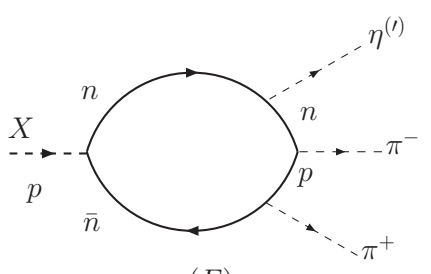

$(F)$

FIG. 3: Diagrams contributing to the strong decay of decay of $X(1835) \rightarrow \eta^{(\prime)} \pi^{+} \pi^{-}$without scalar resonance contribution.

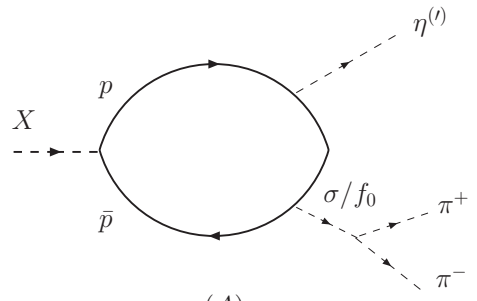

$(A)$

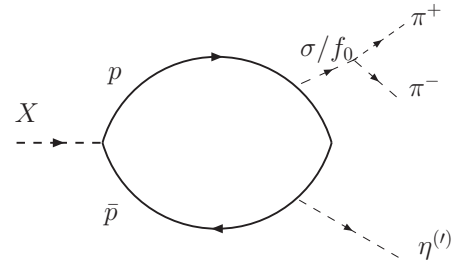

$(C)$

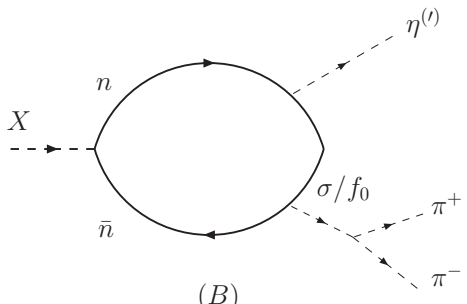

$(B)$

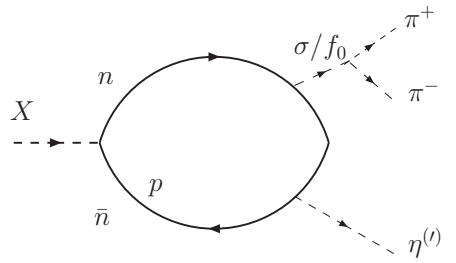

$(D)$

FIG. 4: Diagrams contributing to the strong decay of decay of $X(1835) \rightarrow \eta^{(\prime)} \pi^{+} \pi^{-}$with scalar resonance contribution.

It should be noted that to include the isospin violating effect, the diagrams in Fig. 5 and Fig. 6 should also be considered. For isospin symmetric case the matrix elements for the diagrams of 
Fig. 5 and Fig. 6 have the following relations

$$
i M_{(A)}=-i M_{(B)} ; \quad i M_{(C)}=-i M_{(D)}
$$

In our present work we have considered isospin symmetric case and hence diagrams of Fig. 5 and Fig. 6] do not contribute. In addition, the diagrams with $\rho_{\mu}^{\mp} \eta^{(\prime)} \stackrel{\leftrightarrow}{\partial}_{\mu} \pi^{ \pm}$vertex also have not been considered due to the G-parity conservation.

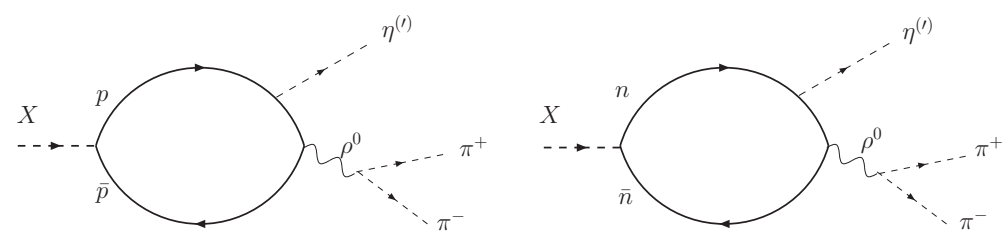

(A)

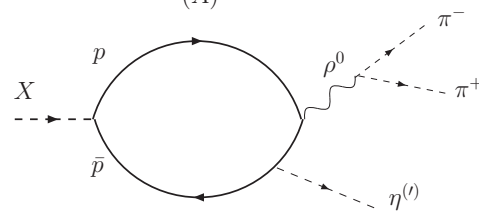

$(C)$
$(B)$

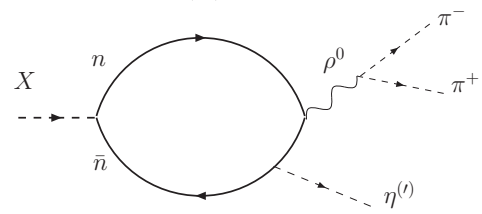

$(D)$

FIG. 5: Diagrams contributing to the strong decay $X(1835) \rightarrow \eta^{(\prime)} \pi^{+} \pi^{-}$from the $\rho$ meson exchange.

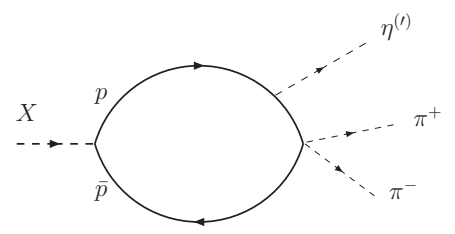

$(A)$

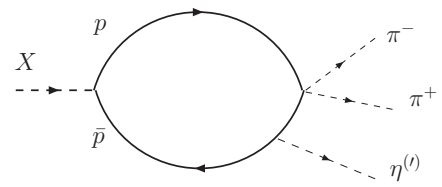

$(C)$

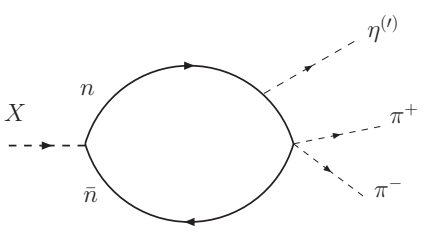

$(B)$

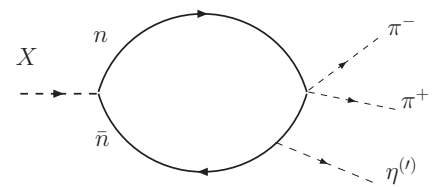

$(D)$

FIG. 6: Diagrams contributing to the strong decay $X(1835) \rightarrow \eta^{(\prime)} \pi^{+} \pi^{-}$from the two-meson-nucleon vertex.

In the following calculation, we label the momenta of the relevant particles according to the scheme $X(p) \rightarrow \pi^{+}\left(q_{1}\right)+\pi^{-}\left(q_{2}\right)+\eta^{(\prime)}\left(q_{3}\right)$. The partial decay width is related to the invariant matrix element $M\left(p \rightarrow q_{1}+q_{2}+q_{3}\right)$ by the relation

$$
\Gamma\left(X(1835) \rightarrow \pi^{+} \pi^{-} \eta^{(\prime)}\right)=\frac{1}{2 m_{X}} \int\left|M^{2}\right| d \Phi
$$


where $d \Phi$ is the Lorentz invariant phase space volume element

$$
d \Phi=(2 \pi)^{4} \delta^{4}\left(p-\sum_{i=1}^{3} q_{i}\right) \prod_{i=1}^{3} \frac{d \mathbf{q}_{i}}{2 E_{i}(2 \pi)^{3}}
$$

with $E_{1}=\sqrt{m_{\pi}^{2}+\mathbf{q}_{1}^{2}}, E_{2}=\sqrt{m_{\pi}^{2}+\mathbf{q}_{2}^{2}}$ and $E_{3}=\sqrt{m_{\eta^{(\prime)}}^{2}+\mathbf{q}_{3}^{2}}$. After integrating the delta function over the solid-angle elements $d \Omega_{1}$ and $d \Omega_{2}$ and treating the $\mathrm{X}(1835)$ as an unpolarized particle, the partial decay width can be expressed as a two dimensional integral

$$
\Gamma\left(X(1835) \rightarrow \eta^{(\prime)} \pi^{+} \pi^{-}\right)=\frac{1}{64 \pi^{3} m_{X}} \int\left|M^{2}\right| d E_{1} d E_{2}
$$

The matrix elements are calculated by evaluating the loop integral. For example, the matrix element $M_{(A)}$ for the corresponding diagram (A) in Fig. 3 is

$$
\begin{aligned}
i M_{(A)}= & -g_{\text {eff }} \frac{2}{(2 m)^{3}} \int \frac{d^{4} k}{(2 \pi)^{4}} \tilde{\Phi}\left(\left(k-\frac{p}{2}\right)\right) \\
& \times \frac{\operatorname{Tr}\left\{(\not k+m) \gamma_{5}[(\not k-\not k)+m] \not \not_{3} \gamma_{5}\left[\left(\not k-\not \not_{1}-\not q_{2}\right)+m\right] \not q_{2} \gamma_{5}\left[\left(\not k-\not q_{1}\right)+m\right] \not q_{1} \gamma_{5}\right\}}{\left(k^{2}-m^{2}\right)\left[(k-p)^{2}-m^{2}\right]\left[\left(k-q_{1}-q_{2}\right)^{2}-m^{2}\right]\left[\left(k-q_{1}\right)^{2}-m^{2}\right]}
\end{aligned}
$$

where $g_{\text {eff }}=g_{X} g_{N \bar{N} \pi}^{2} g_{N \bar{N} \eta^{(\prime)}}$. After performing the trace calculation, the matrix element can be decomposed in terms of the tensor structure

$$
i M_{(A)}=-g_{\text {eff }} \frac{2}{(2 m)^{3}}\left[4 \alpha_{0} D_{0}+4 \alpha_{\mu} D^{\mu}+4 \alpha_{\mu \nu} D^{\mu \nu}+4 \alpha_{\mu \nu \alpha} D^{\mu \nu \alpha}\right]
$$

where $\alpha$ 's are functions of the external momenta and $D$ 's are the loop integrals. Their explicit forms are given in Appendix A.

The results for the decay widths of $\Gamma\left(X \rightarrow \eta^{(\prime)} \pi^{+} \pi^{-}\right)$in the energy region $\Lambda_{X}=2.0-3.0 \mathrm{GeV}$ are

$$
\begin{aligned}
\Gamma\left(X \rightarrow \eta^{\prime} \pi^{+} \pi^{-}\right)=0.580-1.273 \mathrm{MeV} ; & \Gamma^{P}\left(X \rightarrow \eta^{\prime} \pi^{+} \pi^{-}\right)=0.335-0.400 \mathrm{MeV} \\
\Gamma\left(X \rightarrow \eta \pi^{+} \pi^{-}\right)=6.522-13.29 \mathrm{MeV} ; & \Gamma^{P}\left(X \rightarrow \eta \pi^{+} \pi^{-}\right)=1.550-1.926 \mathrm{MeV}
\end{aligned}
$$

where the upper index $P$ means that the results are from the pure pseudoscalar processes illustrated in Fig. 3. The above decay widths increase with increase in $\Lambda_{X}$. To obtain the above results, the coupling constant $g_{X}$ calculated before and the coupling constants given above were used. Using the central value of the total width $\Gamma(X(1835))=67.7 \mathrm{MeV}[1]$, the branching ratios turn out to be

$$
\begin{aligned}
\operatorname{BR}\left(X \rightarrow \eta^{\prime} \pi^{+} \pi^{-}\right) & \simeq 8.57 \times 10^{-3}-1.88 \times 10^{-2} \\
\operatorname{BR}\left(X \rightarrow \eta \pi^{+} \pi^{-}\right) & \simeq 9.63 \times 10^{-2}-1.96 \times 10^{-1}
\end{aligned}
$$


Using the result $\mathrm{BR}(J / \psi \rightarrow \gamma X) \sim(0.5-2) \times 10^{-3}[34]$, the following product for branching fraction is obtained

$$
\operatorname{BR}(J / \psi \rightarrow \gamma X) \operatorname{BR}\left(X \rightarrow \eta^{\prime} \pi^{+} \pi^{-}\right) \simeq(0.428-1.714) \times 10^{-5}-(0.94-3.76) \times 10^{-5}
$$

which is much smaller than the observed data. The uncertainties in the parentheses are from the uncertainty of $\operatorname{BR}(J / \psi \rightarrow \gamma X)$. The large uncertainty comes from the measurement of $\operatorname{BR}(J / \psi \rightarrow$ $\gamma X)$. In addition, the product of branching fraction $\operatorname{BR}(J / \psi \rightarrow \gamma X) \operatorname{BR}\left(X \rightarrow \eta \pi^{+} \pi^{-}\right)$yields

$$
\operatorname{BR}(J / \psi \rightarrow \gamma X) \operatorname{BR}\left(X \rightarrow \eta \pi^{+} \pi^{-}\right) \simeq(0.418-1.926) \times 10^{-4}-(0.963-3.852) \times 10^{-4}
$$

where the uncertainties in the parentheses are also from the uncertainty of $\operatorname{BR}(J / \psi \rightarrow \gamma X)$.

Our calculation shows that the strong decay width $\Gamma\left(X(1835) \rightarrow \eta^{\prime} \pi^{+} \pi^{-}\right)$based on the baryonium assumption in the energy scale $2.0 \mathrm{GeV} \leq \Lambda_{X} \leq 3.0 \mathrm{GeV}$ is much smaller than the data which leads to the conclusion that the $\mathrm{X}(1835)$ may not be a baryonium. In addition, we have also predicted the strong decay width of $\Gamma\left(X \rightarrow \eta \pi^{+} \pi^{-}\right)$should be larger than that of $\Gamma\left(X(1835) \rightarrow \eta^{\prime} \pi^{+} \pi^{-}\right)$if the $\mathrm{X}(1835)$ is a baryonium due to the large phase space and coupling constant $g_{N \bar{N} \eta}$.

\section{B. Radiative decay of $X(1835) \rightarrow 2 \gamma$.}

The $\mathrm{X}(1835)$ state can decay into two photons. Since the $\mathrm{X}(1835)$ state is a pseudoscalar state the radiative decay is an anomalous process. The matrix element can be written as

$$
i M^{\mu \nu}(X(1835) \rightarrow 2 \gamma)=\alpha_{\mathrm{em}} \epsilon_{\mu \nu \alpha \beta} p_{\alpha} q_{\beta} G_{X \gamma \gamma}
$$

where $q$ and $p$ are the momenta of the two final photons. Using the above expression the decay width is given by

$$
\Gamma(X(1835) \rightarrow 2 \gamma)=\frac{1}{8 \pi m_{X}}|M|^{2} \frac{\left|\vec{p}_{c m}\right|}{m_{X}}=\frac{1}{32 \pi} \alpha_{\mathrm{em}}^{2} m_{X}^{3} G_{X \gamma \gamma}^{2}
$$

where $\left|\vec{p}_{\mathrm{cm}}\right|=m_{X} / 2$ is the three-momentum of the decay products.

In our present model, the decay $X(1835) \rightarrow 2 \gamma$ happens via the process given by the diagrams in Fig. 7. Diagrams $(A),(B)$ and their corresponding cross diagrams arise from the gauge of the nonlocal interaction (9). Diagram $(A)$ and its cross one are from quadratic terms of $A_{\mu}$ in the expansion of Eq. (9) while diagram $(B)$ and its cross one are from the linear terms of $A_{\mu}$ and the gauge of the proton free Lagrangian (8). Diagrams $(C)$ and $(D)$ arise from Lagrangian given by (8). 


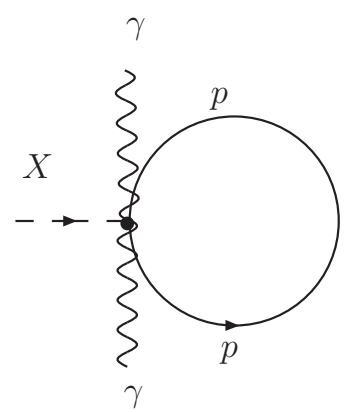

$(A)$

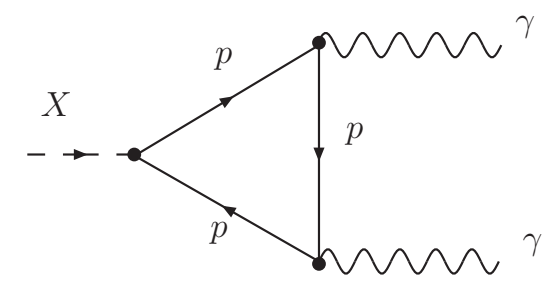

$(C)$

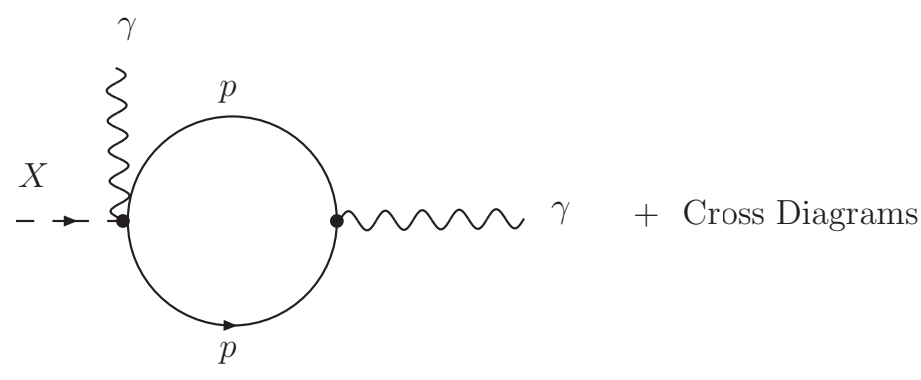

$(B)$

FIG. 7: Diagrams contributing to the radiative decay $X(1835) \rightarrow 2 \gamma$.

From our analysis neither diagram $(A)$ nor diagram $(B)$ contributes to the total matrix element due to the vanishing of the trace of gamma matrices. So we need to calculate only the diagrams $(C)$ and $(D)$ which are the same as that calculated in the triangle anomaly problem. Since the discovery of the triangle anomaly [35, 36], the calculation of these diagrams have been discussed widely in the literature. We had discussed the ambiguities in the calculations induced by regularization, Dirac trace, and momentum shifts [37]. From our calculation

$$
G_{X \gamma \gamma}^{\mathrm{NL}}=g_{X} \frac{2 m_{p}}{\pi \Lambda_{X}^{2}} \int_{0}^{\infty} d \alpha_{1} d \alpha_{2} d \alpha_{3} \frac{1}{\left(1+\alpha_{1}+\alpha_{2}+\alpha_{3}\right)^{2}} \exp \left\{\arg _{\mathrm{em}} / \Lambda_{X}^{2}\right\}
$$

where the upper index NL corresponds to the Nonlocal case and

$$
\arg _{\mathrm{em}}=-\frac{1}{1+\sum_{i=1}^{3} \alpha_{i}}\left(\frac{1}{2}+\alpha_{2}\right)\left(\frac{1}{2}+\alpha_{2}+\alpha_{3}\right) m_{X}^{2}+\left(\frac{1}{4}+\alpha_{2}\right) m_{X}^{2}-\sum_{i=1}^{3} \alpha_{i} m_{p}^{2}
$$

Using the values of the parameters we present the numerical results now. For the effective coupling $G_{X \gamma \gamma}^{\mathrm{NL}}$, and for the scale parameter in the range $2.0 \mathrm{GeV} \leq \Lambda_{X} \leq 3.0 \mathrm{GeV}$, we get the result

$$
G_{X \gamma \gamma}^{\mathrm{NL}}=0.6813 \mathrm{GeV}^{-1}-0.3804 \mathrm{GeV}^{-1}
$$

and the corresponding electromagnetic decay width

$$
\Gamma_{X \gamma \gamma}^{\mathrm{NL}}=1.516 \mathrm{KeV}-0.4726 \mathrm{KeV}
$$




\begin{tabular}{|c|c|r|r|r|c|}
\hline$g_{X}$ & $\Gamma_{X \rightarrow \eta^{\prime} \pi^{+} \pi^{-}}$ & $\Gamma_{X \rightarrow \eta \pi^{+} \pi^{-}}$ & $\Gamma_{X \rightarrow \eta^{\prime} \pi^{0} \pi^{0}}$ & $\Gamma_{X \rightarrow \eta \pi^{0} \pi^{0}}$ & $\Gamma_{X \rightarrow 2 \gamma}$ \\
\hline $2.55-2.37$ & $0.580-1.273$ & $6.522-13.29$ & $0.290-0.637$ & $3.261-6.645$ & $1.516-0.4726$ \\
\hline
\end{tabular}

TABLE I: The $\Lambda_{X}$ dependence of effective coupling constant and decay widths in the region $2.0 \mathrm{GeV} \leq$ $\Lambda_{X} \leq 3.0 \mathrm{GeV}$ (The strong decay width is expressed in unit of $\mathrm{MeV}$ while the electromagnetic decay width is expressed in unit of $\mathrm{KeV}$ ).

Both $G_{X \gamma \gamma}^{\mathrm{NL}}$ and $\Gamma_{X \gamma \gamma}^{\mathrm{NL}}$ decrease with increase in $\Lambda_{X}$.

The radiative decay has been investigated in Ref. [15] treating the $\mathrm{X}(1835)$ as a glueball. The result obtained for the decay width $\Gamma_{X \gamma \gamma}=1.1(0.31-1.1) \mathrm{KeV}$ agrees with our result.

\section{DISCUSSIONS AND CONCLUSIONS}

In this work, the strong decays of $X(1835) \rightarrow \eta^{(\prime)} \pi^{+} \pi^{-}$and electromagnetic decay of $X(1835) \rightarrow$ $2 \gamma$ have been calculated using the effective Lagrangian method. In our work we have treated the $\mathrm{X}(1835)$ as a baryonium. To fix the only free parameter $\Lambda_{X}$ we postulated that the coupling constant $g_{X}$ has to be stable against $\Lambda_{X}$. With this assumption, we varied $\Lambda_{X}$ from $2.0 \mathrm{GeV}$ to $3.0 \mathrm{GeV}$. In the above region the strong decay width of $X(1835) \rightarrow \eta^{\prime} \pi^{+} \pi^{-}$is much smaller than the observed data but our prediction of the electromagnetic decay width of $X(1835) \rightarrow 2 \gamma$ is in agreement with the result where $\mathrm{X}(1835)$ decays through glueball. In addition, we have also calculated the strong decay width $\Gamma\left(X(1835) \rightarrow \eta \pi^{+} \pi^{-}\right)$explicitly. The calculated width is much larger than the partial width of $\Gamma\left(X(1835) \rightarrow \eta^{\prime} \pi^{+} \pi^{-}\right)$which is consistent with the direct analysis of the phase space and the coupling constant.

In the baryonium picture, other decay modes of $\mathrm{X}(1835)$ can also be calculated. Using the isospin relation we get

$$
\begin{aligned}
\Gamma\left(X(1835) \rightarrow \eta^{\prime} \pi^{0} \pi^{0}\right) & =\frac{1}{2} \Gamma\left(X(1835) \rightarrow \eta^{\prime} \pi^{+} \pi^{-}\right)=0.290-0.637 \mathrm{MeV} \\
\Gamma\left(X(1835) \rightarrow \eta \pi^{0} \pi^{0}\right) & =\frac{1}{2} \Gamma\left(X(1835) \rightarrow \eta \pi^{+} \pi^{-}\right)=3.261-6.645 \mathrm{MeV}
\end{aligned}
$$

The other three-pseudoscalar strong decay channels are either isospin symmetry violating processes $\left(\pi^{+} \pi^{-} \pi^{0}\right.$ and $\left.3 \pi^{0}\right)$ or OZI rule suppressed (with Kaon meson in the final state). The four strong decay channels discussed above are dominant among all the three-pseudoscalar channels. We have listed the effective coupling constant $g_{X}$ and their decay widths in the region $2.0 \mathrm{GeV} \leq \Lambda_{X} \leq$ 3.0 GeV in Table. I

It should be noticed that in principal, the finite width effect should be included by introducing 
the Breit-Wigner distribution function. However, this will suppress our results and our final conclusion will not be changed. Moreover, there are also uncertainties from the sigma meson mass and width. Here, we applied the results yielded by unitarizing the $\pi \pi$ and $\pi K$ scattering amplitudes.

To conclude, we have studied the three-pseudoscalar meson and two-photon decays of X(1835). The strong decay width $\Gamma\left(X(1835) \rightarrow \eta^{\prime} \pi^{+} \pi^{-}\right)$is smaller than the experimental data while the two-photon decay width agrees with the result where $\mathrm{X}(1835)$ was assumed to decay via the glueball assumption. From our results $\mathrm{X}(1835)$ cannot be treated as a baryonium, at least in the framework of the composite model as applied in this paper. We have obtained other dominant three-pseudoscalar meson decay channels from the isospin relations. To confirm the structure of $X(1835)$ further theoretical analysis is necessary.

\section{APPENDIX A: DECOMPOSITION OF ONE LOOP INTEGRAL.}

For the one loop integral of diagram (A) of Fig. 3, after performing the trace calculation we get the following decomposition

$$
\begin{aligned}
& i M_{(A)}=-g_{e f f} \frac{2}{(2 m)^{3}} \int \frac{d^{4} k}{(2 \pi)^{4}} \tilde{\Phi}\left(\left(k-\frac{p}{2}\right)\right) \\
& \times \frac{\operatorname{Tr}\left\{(\not k+m) \gamma_{5}[(\not k-\not k)+m] \not q_{3} \gamma_{5}\left[\left(\not k-\not q_{1}-\not q_{2}\right)+m\right] \not q_{2} \gamma_{5}\left[\left(\not k-\not q_{1}\right)+m\right] \not_{1} \gamma_{5}\right\}}{\left(k^{2}-m^{2}\right)\left[(k-p)^{2}-m^{2}\right]\left[\left(k-q_{1}-q_{2}\right)^{2}-m^{2}\right]\left[\left(k-q_{1}\right)^{2}-m^{2}\right]} \\
& =-g_{\text {eff }} \frac{2}{(2 m)^{3}}\left[4 \alpha_{0} D_{0}+4 \alpha_{\mu} D^{\mu}+4 \alpha_{\mu \nu} D^{\mu \nu}+4 \alpha_{\mu \nu \alpha} D^{\mu \nu \alpha}\right]
\end{aligned}
$$

where

$$
\begin{aligned}
\alpha^{0}= & m^{3}\left[p \cdot q_{3} q_{1} \cdot q_{2}-p \cdot q_{2} q_{1} \cdot q_{3}-2 q_{1} \cdot q_{2} q_{1} \cdot q_{3}-q_{1} \cdot q_{3} q_{2}^{2}+p \cdot q_{1} q_{2} \cdot q_{3}+2 q_{1}^{2} q_{2} \cdot q_{3}\right] \\
& +m\left[-p \cdot q_{3} q_{1} \cdot q_{2} q_{1}^{2}-p \cdot q_{2} q_{1} \cdot q_{3} q_{1}^{2}-p \cdot q_{3} q_{1}^{2} q_{2}^{2}+p \cdot q_{1} q_{2} \cdot q_{3} q_{1}^{2}\right] \\
\alpha^{\mu}= & m^{3}\left[q_{1} \cdot q_{2} q_{3}^{\mu}-2 q_{2} \cdot q_{3} q_{1}^{\mu}\right] \\
& +m\left[-2 p \cdot q_{2} q_{1}^{2} q_{3}^{\mu}+4 p \cdot q_{3} q_{1}^{2} q_{2}^{\mu}+4 p \cdot q_{1} q_{1} \cdot q_{2} q_{3}^{\mu}-2 p \cdot q_{3} q_{1} \cdot q_{2} q_{1}^{\mu}+2 p \cdot q_{2} q_{1} \cdot q_{3} q_{1}^{\mu}\right. \\
\alpha^{\mu \nu}= & m\left[-4 p \cdot q_{1} q_{2}^{\mu} q_{3}^{\nu}+4 p \cdot q_{2} q_{1}^{\mu} q_{3}^{\nu}-4 p \cdot q_{3} q_{1}^{\mu} q_{2}^{\nu}+4 q_{1} \cdot q_{3} p^{\mu} q_{2}^{\nu}-4 q_{2} \cdot q_{3} p^{\mu} q_{1}^{\nu}\right] \\
& +m\left[3 p \cdot q_{3} q_{1} \cdot q_{2}-3 p \cdot q_{2} q_{1} \cdot q_{3}+2 q_{1} \cdot q_{2} q_{1} \cdot q_{3}+q_{1} \cdot q_{3} q_{2}^{2}+3 p \cdot q_{1} q_{2} \cdot q_{3}-2 q_{1}^{2} q_{2} \cdot q_{3}\right] g^{\mu \nu} \\
\alpha^{\mu \nu \alpha}= & 2 m\left[q_{2} \cdot q_{3} q_{1}^{\alpha}-q_{1} \cdot q_{2} q_{3}^{\alpha}\right] g^{\mu \nu}
\end{aligned}
$$


and

$$
D_{\{0 ; \mu ; \mu \nu ; \mu \nu \alpha\}}=\int \frac{d^{4} k}{(2 \pi)^{4}} \tilde{\Phi}\left(\left(k-\frac{p}{2}\right)\right) \frac{\left\{1 ; k_{\mu} ; k_{\mu} k_{\nu} ; k_{\mu} k_{\nu} k_{\alpha}\right\}}{\left(k^{2}-m^{2}\right)\left[(k-p)^{2}-m^{2}\right]\left[\left(k-q_{1}-q_{2}\right)^{2}-m^{2}\right]\left[\left(k-q_{1}\right)^{2}-m^{2}\right]}
$$

It is to be noted that due to the relation

$$
\begin{aligned}
k \cdot p & =-\frac{1}{2}\left\{\left[(k-p)^{2}-m^{2}\right]-\left(k^{2}-m^{2}\right)-p^{2}\right\} \\
k \cdot q_{1} & =-\frac{1}{2}\left\{\left[\left(k-q_{1}\right)^{2}-m^{2}\right]-\left(k^{2}-m^{2}\right)-q_{1}^{2}\right\} \\
k \cdot q_{3} & =-\frac{1}{2}\left\{\left[(k-p)^{2}-m^{2}\right]-\left[\left(k-q_{1}-q_{2}\right)^{2}-m^{2}\right]+\left(q_{1}+q_{2}\right)^{2}\right\}
\end{aligned}
$$

the above vector, two- and three- rank four-point integrals can be expressed in terms of scalar four-point and three-point integrals

$$
\begin{array}{r}
\alpha_{\mu} D^{\mu}=\beta_{0}^{V} D_{0}+\beta_{234}^{V} C_{234 ; 0}+\beta_{134}^{V} C_{134 ; 0}+\beta_{124}^{V} C_{124 ; 0}+\beta_{123}^{V} C_{123 ; 0} \\
\alpha_{\mu \nu} D^{\mu \nu}=m^{3} \beta_{0}^{T 1} D_{0}+m \beta_{0}^{T 1} C_{234 ; 0}+m\left[\beta_{123}^{T 1 ; \mu} C_{123 ; \mu}+\beta_{124}^{T 1 ; \mu} C_{124 ; \mu}+\beta_{134}^{T 1 ; \mu} C_{134 ; \mu}+\beta_{234}^{T 1 ; \mu} C_{234 ; \mu}\right] \\
+m\left[\beta_{0}^{T 2} D_{0}+\beta_{123}^{T 2} C_{123 ; 0}+\beta_{124}^{T 2} C_{124 ; 0}+\beta_{234}^{T 2} C_{234 ; 0}\right]
\end{array}
$$

with

$$
\begin{aligned}
& \beta_{0}^{V}=-\frac{1}{2}\left\{m^{3}\left[q_{1} \cdot q_{2}\left[\left(q_{1}+q_{2}\right)^{2}-q^{2}\right]+2 q_{2} \cdot q_{3} q_{1}^{2}\right]\right. \\
& +m\left[-2 q \cdot q_{2} q_{1}^{2}\left[\left(q_{1}+q_{2}\right)^{2}-q^{2}\right]+4 q \cdot q_{3} q_{1}^{2}\left[q_{1}^{2}-\left(q_{1}+q_{2}\right)^{2}\right]\right. \\
& +4 q \cdot q_{1} q_{1} \cdot q_{2}\left[\left(q_{1}+q_{2}\right)^{2}-q^{2}\right]+2 q \cdot q_{3} q_{1} \cdot q_{2} q_{1}^{2}-2 q \cdot q_{2} q_{1} \cdot q_{3} q_{1}^{2} \\
& +4 q_{1} \cdot q_{2} q_{1} \cdot q_{3} q^{2}+2 q \cdot q_{1} q_{2}^{2}\left[\left(q_{1}+q_{2}\right)^{2}-q^{2}\right]+2 q_{1} \cdot q_{3} q_{2}^{2} q^{2}+2 q \cdot q_{1} q_{2} \cdot q_{3} q_{1}^{2} \\
& \left.\left.-2 q_{2} \cdot q_{3} q_{1}^{2} q^{2}\right]\right\} \\
& \beta_{234}^{V}=-\frac{1}{2}\left\{2 m^{3} q_{2} \cdot q_{3}+m\left[2 q \cdot q_{3} q_{1} \cdot q_{2}-2 q \cdot q_{2} q_{1} \cdot q_{3}+4 q_{1} \cdot q_{2} q_{1} \cdot q_{3}+2 q_{1} \cdot q_{3} q_{2}^{2}+2 q \cdot q_{1} q_{2} \cdot q_{3}\right.\right. \\
& \left.\left.-2 q_{2} \cdot q_{3} q_{1}^{2}\right]\right\} \\
& \beta_{134}^{V}=-\frac{1}{2}\left\{m^{3} q_{1} \cdot q_{2}+m\left[-2 q \cdot q_{2} q_{1}^{2}+4 q \cdot q_{1} q_{1} \cdot q_{2}-4 q_{1} \cdot q_{2} q_{1} \cdot q_{3}+2 q \cdot q_{1} q_{2}^{2}-2 q_{1} \cdot q_{3} q_{2}^{2}\right.\right. \\
& \left.\left.+2 q_{2} \cdot q_{3} q_{1}^{2}\right]\right\} \\
& \beta_{124}^{V}=-\frac{1}{2}\left\{-m^{3} q_{1} \cdot q_{2}+m\left[2 q \cdot q_{2} q_{1}^{2}+4 q \cdot q_{3} q_{1}^{2}-4 q \cdot q_{1} q_{1} \cdot q_{2}-2 q \cdot q_{1} q_{2}^{2}\right]\right\}
\end{aligned}
$$




$$
\begin{aligned}
\beta_{123}^{V}= & -\frac{1}{2}\left\{-2 m^{3} q_{2} \cdot q_{3}+m\left[-4 q \cdot q_{3} q_{1}^{2}-2 q \cdot q_{3} q_{1} \cdot q_{2}+2 q \cdot q_{2} q_{1} \cdot q_{3}-2 q \cdot q_{1} q_{2} \cdot q_{3}\right]\right\} \\
\beta_{0}^{T 1}= & 3 p \cdot q_{3} q_{1} \cdot q_{2}-3 p \cdot q_{2} q_{1} \cdot q_{3}+2 q_{1} \cdot q_{2} q_{1} \cdot q_{3}+q_{1} \cdot q_{3} q_{2}^{2}+3 p \cdot q_{1} q_{2} \cdot q_{3}-2 q_{1}^{2} q_{2} \cdot q_{3} \\
\beta_{123 ; \mu}^{T 1}= & 2 p \cdot q_{3} q_{2}^{\mu} \\
\beta_{124 ; \mu}^{T 1}= & 2 p \cdot q_{2} q_{1}^{\mu}-2 p \cdot q_{1} q_{2}^{\mu} \\
\beta_{134 ; \mu}^{T 1}= & 2 p \cdot q_{1} q_{2}^{\mu}-2 p \cdot q_{2} q_{1}^{\mu}-2 q_{1} \cdot q_{3} q_{2}^{\mu}+2 q_{2} \cdot q_{3} q_{1}^{\mu} \\
\beta_{234 ; \mu}^{T 1}= & 2 q_{1} \cdot q_{3} q_{2}^{\mu}-2 p \cdot q_{3} q_{2}^{\mu}-2 q_{2} \cdot q_{3} q_{1}^{\mu} \\
\beta_{0}^{T 2}= & -\frac{1}{2}\left\{2 q \cdot q_{1}\left[\left(q_{1}+q_{2}\right)^{2}-q^{2}\right]\left[-\left(q_{1}+q_{2}\right)^{2}+q_{1}^{2}\right]+2 q \cdot q_{2} q_{1}^{2}\left[\left(q_{1}+q_{2}\right)^{2}-q^{2}\right]\right. \\
& \left.-2 q \cdot q_{3} q_{1}^{2}\left[-\left(q_{1}+q_{2}\right)^{2}+q_{1}^{2}\right]+2 q_{1} \cdot q_{3} q^{2}\left[-\left(q_{1}+q_{2}\right)^{2}+q_{1}^{2}\right]+2 q_{2} \cdot q_{3} q^{2} q_{1}^{2}\right\} \\
\beta_{123}^{T 2}= & -\frac{1}{2}\left\{-2 q \cdot q_{1}\left[\left(q_{1}+q_{2}\right)^{2}-q^{2}\right]-2 q \cdot q_{2}\left[\left(q_{1}+q_{2}\right)^{2}-q^{2}\right]\right. \\
\left.\quad+2 q \cdot q_{3} q_{1}^{2}-2 q_{1} \cdot q_{3} q^{2}-2 q_{2} \cdot q_{3} q^{2}\right\} & \\
\beta_{124}^{T 2}= & -\frac{1}{2}\left\{2 q \cdot q_{1}\left[\left(q_{1}+q_{2}\right)^{2}-q^{2}\right]-2 q \cdot q_{3} q_{1}^{2}+2 q_{1} \cdot q_{3} q^{2}\right\} \\
\beta_{234}^{T 2}= & -\frac{1}{2}\left\{2 q \cdot q_{2}\left[\left(q_{1}+q_{2}\right)^{2}-q^{2}\right]+2 q_{2} \cdot q_{3} q^{2}\right\}
\end{aligned}
$$

and

$$
\begin{aligned}
C_{123 ;\{0 ; \mu\}} & =\int \frac{d^{4} k}{(2 \pi)^{4}} \tilde{\Phi}\left(\left(k-\frac{p}{2}\right)\right) \frac{\left\{1 ; k_{\mu}\right\}}{\left(k^{2}-m^{2}\right)\left[(k-p)^{2}-m^{2}\right]\left[\left(k-q_{1}-q_{2}\right)^{2}-m^{2}\right]} \\
C_{124 ;\{0 ; \mu\}} & =\int \frac{d^{4} k}{(2 \pi)^{4}} \tilde{\Phi}\left(\left(k-\frac{p}{2}\right)\right) \frac{\left\{1 ; k_{\mu}\right\}}{\left(k^{2}-m^{2}\right)\left[(k-p)^{2}-m^{2}\right]\left[\left(k-q_{1}\right)^{2}-m^{2}\right]} \\
C_{234 ;\{0 ; \mu\}} & =\int \frac{d^{4} k}{(2 \pi)^{4}} \tilde{\Phi}\left(\left(k-\frac{p}{2}\right)\right) \frac{\left\{1 ; k_{\mu}\right\}}{\left[(k-p)^{2}-m^{2}\right]\left[\left(k-q_{1}-q_{2}\right)^{2}-m^{2}\right]\left[\left(k-q_{1}\right)^{2}-m^{2}\right]} \\
C_{134 ;\{0 ; \mu\}} & =\int \frac{d^{4} k}{(2 \pi)^{4}} \tilde{\Phi}\left(\left(k-\frac{p}{2}\right)\right) \frac{\left\{1 ; k_{\mu}\right\}}{\left(k^{2}-m^{2}\right)\left[\left(k-q_{1}-q_{2}\right)^{2}-m^{2}\right]\left[\left(k-q_{1}\right)^{2}-m^{2}\right]}
\end{aligned}
$$

Using the above, the matrix element $i M_{(A)}$ can be expressed in terms of the scalar, and vector $C$ and $D$ functions. For the scalar, vector $C$ and $D$ functions one can evaluate the momentum integral explicitly and yield the following results.

$$
\begin{aligned}
D_{\{0 ; \mu\}} & =\frac{i}{16 \pi^{2}} \frac{1}{\Lambda_{X}^{4}} \int_{0}^{\infty} d \alpha_{1} d \alpha_{2} d \alpha_{3} d \alpha_{4} \frac{1}{\left(1+\tilde{\alpha}_{4}\right)^{2}}\left\{1 ; \frac{1}{1+\tilde{\alpha}_{4}} P_{D ; \mu}\right\} \exp \left\{\arg _{D} / \Lambda_{X}^{2}\right\} \\
C_{123 ;\{0 ; \mu\}} & =-\frac{i}{16 \pi^{2}} \frac{1}{\Lambda_{X}^{2}} \int_{0}^{\infty} d \alpha_{1} d \alpha_{2} d \alpha_{3} \frac{1}{\left(1+\tilde{\alpha}_{3}\right)^{2}}\left\{1 ; \frac{1}{1+\tilde{\alpha}_{3}} P_{C_{123} ; \mu}\right\} \exp \left\{\arg _{C_{123}} / \Lambda_{X}^{2}\right\} \\
C_{124 ;\{0 ; \mu\}} & =-\frac{i}{16 \pi^{2}} \frac{1}{\Lambda_{X}^{2}} \int_{0}^{\infty} d \alpha_{1} d \alpha_{2} d \alpha_{3} \frac{1}{\left(1+\tilde{\alpha}_{3}\right)^{2}}\left\{1 ; \frac{1}{1+\tilde{\alpha}_{3}} P_{C_{124} ; \mu}\right\} \exp \left\{\arg _{C_{124}} / \Lambda_{X}^{2}\right\} \\
C_{234 ;\{0 ; \mu\}} & =-\frac{i}{16 \pi^{2}} \frac{1}{\Lambda_{X}^{2}} \int_{0}^{\infty} d \alpha_{1} d \alpha_{2} d \alpha_{3} \frac{1}{\left(1+\tilde{\alpha}_{3}\right)^{2}}\left\{1 ; \frac{1}{1+\tilde{\alpha}_{3}} P_{C_{234} ; \mu}\right\} \exp \left\{\arg _{C_{234}} / \Lambda_{X}^{2}\right\}
\end{aligned}
$$




$$
C_{134 ;\{0 ; \mu\}}=-\frac{i}{16 \pi^{2}} \frac{1}{\Lambda_{X}^{2}} \int_{0}^{\infty} d \alpha_{1} d \alpha_{2} d \alpha_{3} \frac{1}{\left(1+\tilde{\alpha}_{3}\right)^{2}}\left\{1 ; \frac{1}{1+\tilde{\alpha}_{3}} P_{C_{134} ; \mu}\right\} \exp \left\{\arg _{C_{134}} / \Lambda_{X}^{2}\right\}
$$

where

$$
\begin{aligned}
\tilde{\alpha}_{n} & =\sum_{i=1}^{n} \alpha_{i} \\
P_{D ; \mu} & =\left(\frac{1}{2}+\alpha_{2}\right) p_{\mu}+\alpha_{3} q_{1 ; \mu}+\alpha_{4}\left(q_{1}+q_{2}\right)_{\mu} \\
P_{C_{123} ; \mu} & =\left(\frac{1}{2}+\alpha_{2}\right) p_{\mu}+\alpha_{3}\left(q_{1}+q_{2}\right)_{\mu} \\
P_{C_{124} ; \mu} & =\left(\frac{1}{2}+\alpha_{2}\right) p_{\mu}+\alpha_{3} q_{1 ; \mu} \\
P_{C_{234} ; \mu} & =\left(\frac{1}{2}+\alpha_{1}\right) p_{\mu}+\alpha_{2}\left(q_{1}+q_{2}\right)_{\mu}+\alpha_{3} q_{1 ; \mu} \\
P_{C_{134} ; \mu} & =\frac{1}{2} p_{\mu}+\alpha_{2}\left(q_{1}+q_{2}\right)_{\mu}+\alpha_{3} q_{1 ; \mu} \\
\arg _{D} & =-\frac{1}{1+\tilde{\alpha}_{4}} P_{D}^{2}+\left(\frac{1}{4}+\alpha_{2}\right) p^{2}+\alpha_{3} q_{1}^{2}+\alpha_{4}\left(q_{1}+q_{2}\right)^{2}-\tilde{\alpha}_{4} m^{2} \\
\arg _{C_{123}} & =-\frac{1}{1+\tilde{\alpha}_{3}} P_{C_{123}}^{2}+\left(\frac{1}{4}+\alpha_{2}\right) p^{2}+\alpha_{3}\left(q_{1}+q_{2}\right)^{2}-\tilde{\alpha}_{3} m^{2} \\
\arg _{C_{124}} & =-\frac{1}{1+\tilde{\alpha}_{3}} P_{C_{124}}^{2}+\left(\frac{1}{4}+\alpha_{2}\right) p^{2}+\alpha_{3} q_{1}^{2}-\tilde{\alpha}_{3} m^{2} \\
\arg _{C_{234}} & =-\frac{1}{1+\tilde{\alpha}_{3}} P_{C_{234}}^{2}+\left(\frac{1}{4}+\alpha_{1}\right) p^{2}+\alpha_{2}\left(q_{1}+q_{2}\right)^{2}+\alpha_{3} q_{1}^{2}-\tilde{\alpha}_{3} m^{2} \\
\arg _{C_{134}} & =-\frac{1}{1+\tilde{\alpha}_{3}} P_{C_{134}}^{2}+\frac{1}{4} p^{2}+\alpha_{2}\left(q_{1}+q_{2}\right)^{2}+\alpha_{3} q_{1}^{2}-\tilde{\alpha}_{3} m^{2}
\end{aligned}
$$

\section{ACKNOWLEDGMENTS}

I would like to thanks Profs. Amand Faessler, Thomas Gutsche and Yu-Peng Yan for valuable discussions we had with them. We also thank Prof. Yue-Liang Wu(ITP, CAS) for suggesting the problem. This work was supported by International Graduiertenkolleg der DFG GRK683 "Hadronen im Vakuum, in Kernen und in Sternen".

[1] M. Ablikim et al. [BES Collaboration], Phys. Rev. Lett. 95, 262001 (2005) arXiv:hep-ex/0508025.

[2] J. Z. Bai et al. [BES Collaboration], Phys. Rev. Lett. 91, 022001 (2003) arXiv:hep-ex/0303006.

[3] J. L. Rosner, AIP Conf. Proc. 815, 218 (2006) arXiv:hep-ph/0508155.

[4] J. L. Rosner, Phys. Rev. D 68, 014004 (2003) arXiv:hep-ph/0303079].

[5] A. Datta and P. J. O’Donnell, Phys. Lett. B 567, 273 (2003) arXiv:hep-ph/0306097.

[6] B. S. Zou and H. C. Chiang, Phys. Rev. D 69, 034004 (2004) arXiv:hep-ph/0309273.

[7] X. a. Liu, X. Q. Zeng, Y. B. Ding, X. Q. Li, H. Shen and P. N. Shen, arXiv:hep-ph/0406118. 
[8] C. H. Chang and H. R. Pang, Commun. Theor. Phys. 43, 275 (2005) arXiv:hep-ph/0407188.

[9] A. Sibirtsev, J. Haidenbauer, S. Krewald, U. G. Meissner and A. W. Thomas, Phys. Rev. D 71, 054010 (2005) arXiv:hep-ph/0411386.

[10] M. L. Yan, S. Li, B. Wu and B. Q. Ma, Phys. Rev. D 72, 034027 (2005).

[11] G. J. Ding and M. L. Yan, Phys. Rev. C 72, 015208 (2005) arXiv:hep-ph/0502127.

[12] S. L. Zhu and C. S. Gao, Commun. Theor. Phys. 46, 291 (2006) arXiv:hep-ph/0507050.

[13] Z. G. Wang and S. L. Wan, J. Phys. G 34, 505 (2007) arXiv:hep-ph/0601105.

[14] N. Kochelev and D. P. Min, Phys. Lett. B 633, 283 (2006) arXiv:hep-ph/0508288.

[15] B. A. Li, Phys. Rev. D 74, 034019 (2006) arXiv:hep-ph/0510093.

[16] X. G. He, X. Q. Li, X. Liu and J. P. Ma, Eur. Phys. J. C 49, 731 (2007) arXiv:hep-ph/0509140.

[17] T. Huang and S. L. Zhu, Phys. Rev. D 73, 014023 (2006) arXiv:hep-ph/0511153.

[18] E. Klempt and A. Zaitsev, Phys. Rept. 454, 1 (2007) arXiv:0708.4016 [hep-ph]].

[19] D. M. Li and B. Ma, Phys. Rev. D 77, 074004 (2008) arXiv:0801.4821 [hep-ph]].

[20] S. Weinberg, Phys. Rev. 130, 776 (1963);

[21] A. Salam, Nuovo Cim. 25, 224 (1962);

[22] K. Hayashi, M. Hirayama, T. Muta, N. Seto and T. Shirafuji, Fortsch. Phys. 15, 625 (1967).

[23] A. Faessler, T. Gutsche, V. E. Lyubovitskij and Y. L. Ma, Phys. Rev. D 76, 014005 (2007) arXiv:0705.0254 [hep-ph]].

[24] A. Faessler, T. Gutsche, V. E. Lyubovitskij and Y. L. Ma, Phys. Rev. D 76, 114008 (2007) arXiv:0709.3946 [hep-ph]].

[25] Y. b. Dong, A. Faessler, T. Gutsche and V. E. Lyubovitskij, arXiv:0802.3610 [hep-ph].

[26] A. Faessler, T. Gutsche, V. E. Lyubovitskij and Y. L. Ma, arXiv:0801.2232 [hep-ph].

[27] W. M. Yao et al. [Particle Data Group], J. Phys. G 33 (2006) 1.

[28] A. M. Abdel-Rehim, D. Black, A. H. Fariborz and J. Schechter, Phys. Rev. D 67, 054001 (2003) arXiv:hep-ph/0210431.

[29] R. Sinha and S. Okubo, Phys. Rev. D 30, 2333 (1984).

[30] W. H. Liang, P. N. Shen, B. S. Zou and A. Faessler, Eur. Phys. J. A 21, 487 (2004) arXiv:nucl-th/0404024.

[31] R. Machleidt, K. Holinde and C. Elster, Phys. Rept. 149 (1987) 1.

[32] S. Mandelstam, Annals Phys. 19, 25 (1962).

[33] J. Terning, Phys. Rev. D 44, 887 (1991).

[34] S. Jin, talk presented at the International Conference on QCD and Hadronic Physics, Beijing, China, 6/16-6/60,2005; S. S. Fang, talk presented at the International Conference on QCD and Hadronic Physics, Beijing, China, 6/16-6/60,2005; X. Y. Shen, talk presented at Lepton-Photon 2005, 6/307/5, 2005, Uppsala, Sweden.

[35] J. S. Bell and R. Jackiw, Nuovo Cim. A 60 (1969) 47.

[36] S. L. Adler, Phys. Rev. 177 (1969) 2426. 
[37] Y. L. Ma and Y. L. Wu, Int. J. Mod. Phys. A 21, 6383 (2006) arXiv:hep-ph/0509083. 